\title{
Iterations of rational functions: which hyperbolic components contain polynomials?
}

\author{
by \\ Feliks Przyt y cki (Warszawa) \\ Dedicated to the memory of Wiestaw Szlenk, \\ my teacher and friend
}

\begin{abstract}
Let $H^{d}$ be the set of all rational maps of degree $d \geq 2$ on the Riemann sphere, expanding on their Julia set. We prove that if $f \in H^{d}$ and all, or all but one, critical points (or values) are in the basin of immediate attraction to an attracting fixed point then there exists a polynomial in the component $H(f)$ of $H^{d}$ containing $f$. If all critical points are in the basin of immediate attraction to an attracting fixed point or a parabolic fixed point then $f$ restricted to the Julia set is conjugate to the shift on the one-sided shift space of $d$ symbols. We give exotic examples of maps of an arbitrary degree $d$ with a non-simply connected completely invariant basin of attraction and arbitrary number $k \geq 2$ of critical points in the basin. For such a map $f \in H^{d}$ with $k<d$ there is no polynomial in $H(f)$. Finally, we describe a computer experiment joining an exotic example to a Newton's method rational function (for a polynomial) with a 1-parameter family of rational maps.
\end{abstract}

Introduction. In the space $Q^{d}$ of rational maps of degree $d \geq 2$ of the Riemann sphere $\overline{\mathbb{C}}$ denote by $H^{d}$ the set of maps which are expanding on their Julia set. Expanding means that there exists $n>0$ such that for every $z$ in the Julia set, $\left|\left(f^{n}\right)^{\prime}(z)\right|>1$, derivative in the standard spherical Riemann metric. We call $z \in \overline{\mathbb{C}}$ a critical point if $f^{\prime}(z)=0$. We call $v$ a critical value if $v=f(z)$ for a critical point $z$.

In Section 1 we prove the following:

Theorem A. Let $f \in H^{d}$. Suppose that all, or all but one, critical values of $f$ are in a basin $B(f)$ of immediate attraction to one attracting

1991 Mathematics Subject Classification: Primary 58F23.

This is a revised and extended version of a manuscript "Polynomials in hyperbolic components", IMS SUNY at Stony Brook, June 1992, and the IMS Stony Brook preprint 1994/6.

Research partially supported by Polish KBN Grants 210469101 "Iteracje i Fraktale" and 210909101 “...Układy Dynamiczne". 
f-fixed point $p$. Then the component $H(f)$ of $H^{d}$ containing $f$ also contains a polynomial.

(The critical values in Theorem A are counted without multiplicities. However, throughout the paper, critical points are counted with multiplicities.)

COROLlary B. If all critical points of $f$ are in $B(f)$ then $f$ restricted to the Julia set $J(f)$ is conjugate to the full one-sided shift.

Theorem C. Let $f \in Q^{d}$, $p$ be a parabolic fixed point and $B(f)$ be a basin of immediate attraction to $p$ adjacent to $p$ such that $f(B(f))=B(f)$ (which is equivalent to $f^{\prime}(p)=1$ ). Suppose that all critical points of $f$ are in $B(f)$. Then $f$ restricted to $J(f)$ is conjugate to the full one-sided shift.

The proof of Theorem C is similar to that of Corollary B so it will only be sketched.

It is much easier to prove that, under the assumptions of Corollary B or Theorem C, $J(f)$ is Cantor, $\left.f\right|_{J(f)}$ is conjugate to a topological Markov chain and $\left.f^{n}\right|_{J(f)}$ is conjugate to a 1-sided shift for some $n>0$, than to prove that $\left.f\right|_{J(f)}$ itself is conjugate to a full 1-sided shift. See Remarks 1.5 and 1.6.

The questions answered in Corollary B and Theorem $\mathrm{C}$ were posed to me by John Milnor. In the case of the basin of a sink he suggested to join critical values to the sink along trajectories of the gradient flow, Morse curves, described below. This was a fruitful idea. After proving Theorem A and Corollary B, I learned that these facts were proved by P. Makienko $\left(^{1}\right)$ in his $\mathrm{PhD}$ paper but stayed unpublished $\left({ }^{1}\right)$. Corollary B is also stated in [GK] but proved only for $d=2$.

Note that Corollary B and Theorem $\mathrm{C}$ depend on holomorphic phenomena. Indeed, there exists a 1-sided topological Markov chain $T$ with each point having 10 preimages which has the same $\zeta$-function as the full 1 -sided shift $S_{10}$ on 10 symbols and whose sufficiently high power $T^{n}$ is conjugate to $S_{10}^{n}$, but $T$ itself is not conjugate to $S_{10}$ [Boy].

If $B$, the immediate basin of attraction to an attracting fixed point, is simply connected, then the number of critical points of $f$ in $B$ is equal to $\operatorname{deg}\left(\left.f\right|_{B}\right)-1$ (because $f$ pulled back to the unit disc $\mathbb{D}$ by a Riemann mapping is a Blaschke product which has $\operatorname{deg}\left(\left.f\right|_{B}\right)-1$ critical points in $\mathbb{D}$, and the same number outside).

If $B$ is the basin of attraction to $\infty$ for a polynomial and $B$ is not simply connected, then the number of critical points of $f$ in $B$ is at least $\operatorname{deg}\left(\left.f\right|_{B}\right)$ (including $\infty$ as a $(\operatorname{deg}(f)-1)$-multiple critical point).

$\left({ }^{1}\right)$ Recently his proof appeared in the preprint $[\mathrm{M}]$. It influenced our revised version of the paper. Also our Theorem $\mathrm{C}$ is proved in $[\mathrm{M}]$. 
Surprisingly this is false in general. The "proof" that if the basin is not simply connected then it contains at least as many critical points as the degree of $f$ on it, given in $[\mathrm{P}]$, is erroneous for degree larger than 2: the corresponding Lemma in $[\mathrm{P}]$ is false.

In Section 2, with the use of the quasiconformal surgery technique [D], we prove the existence of exotic basins:

THEOREM D. There exists a rational function $f$ of an arbitrary degree $d \geq 3$ with a completely invariant (i.e. invariant under $f^{-1}$ ) non-simply connected basin of immediate attraction to an attracting fixed point, and with an arbitrary number $k, 2 \leq k \leq 2 d-2$, of critical points in the basin.

The conclusion is that the assumption that all, or all but one, critical values are in the basin in Theorem A, is essential. Namely, for $k \leq d-1$ Theorem D yields

Corollary E. For every $d \geq 3$ there exists $f \in H^{d}$ with a completely invariant basin of immediate attraction to an attracting fixed point such that $H(f)$ contains no polynomial.

Neither in Theorem A nor in Corollary E does it matter that $f \in H^{d}$. Only the basin $B(f)$ matters. The reader will find appropriate precise assertions in Sections 1 and 2.

I checked with computer that $f(z)=z^{2}+c+b /(z-a)$ for $c=-3.121092$, $a=1.719727$ and $b=.3142117$ is an exotic example for $d=3$ whose existence is asserted in Corollary E. I am grateful to Ben Bielefeld and Scott Sutherland for their help in producing several computer pictures of Julia sets for such $f$ 's and related pictures in the parameter space. Ben invented the parametrization in which the pictures were done. Our 1-parameter families join exotic examples of the above type with $d=3$, having two superattracting fixed points and a critical point of period 2, to functions having three superattracting fixed points. It is easy to see (and well known) that the latter functions must be Newton's method rational functions for degree 3 polynomials.

(If $f \in Q^{d}$ has $d$ superattracting fixed points then in appropriate holomorphic coordinates on $\overline{\mathbb{C}}$ it is Newton's for a degree $d$ polynomial. Hint to the proof: Change first the coordinates on $\overline{\mathbb{C}}$ by a homography so that the unique repelling fixed point becomes $\infty$.)

\section{Rearranging critical values. Proofs of Theorem A and Corol-} lary B. Let $B(f)$ be the immediate basin of attraction to an attracting fixed point $p$ for a rational map $f \in H^{d}$. Suppose $f^{\prime}(p) \neq 0$.

In this section we shall make the following types of perturbation of such maps in $H^{d}$ : 
1. Perturbation along a curve $\gamma$. We have in mind the following construction: Suppose there is a curve $\gamma=\gamma(t), t \in[0,1]$, embedded in $B(f)$ with $p \notin \gamma$. Take a small neighbourhood $U$ of $\gamma, U \subset B(f)$, disjoint from a neighbourhood of $p$. Let $g_{t}$ for $t \in[0,1]$ be a diffeomorphism of $\overline{\mathbb{C}}$ so that $g_{t}(\gamma(0))=\gamma(t), g_{t}$ is the identity outside $U, g_{0}=$ id and $g_{t}$ smoothly depends on $t$. We obtain the homotopy $h_{t}=g_{t} \circ f$. Note that though we are talking about a perturbation "along $\gamma$ " we change the map in a neighbourhood of $f^{-1}(\gamma)$.

If the following assumption holds:

$$
p \notin U, \quad h_{t}^{n}(U) \rightarrow p,
$$

then of course the basin of attraction to $p$ is the same for $h_{t}$ as for $f$.

Then we construct an invariant measurable $L^{\infty}$ conformal structure for each $h_{t}$ as follows: We take the standard structure on a small neighbourhood of $p$ and then pull it back by $h_{t}^{-n}$. On the complement we take the standard structure. Now we integrate this structure (we refer to the Measurable Riemann Mapping Theorem $[\mathrm{B}],[\mathrm{AB}])$, and $h_{t}$ in the new coordinates gives a homotopy $f_{t}$ through maps in $H^{d}$. See [D] for this technique.

2. Small $C^{1}$-perturbation. If a map $g$ is $C^{1}$ close to $f$ on $U$ such that cl $U \subset B(f) \backslash\{p\}, f=g$ outside $U$, and near critical points, $g$ differs from $f$ only by affine parts, then clearly $g$ is homotopic to $f$ also through small perturbations satisfying the same conditions as $g$. The condition (1) holds automatically. As before we introduce new conformal structures, integrate them and obtain a perturbation homotopic to $f$ through maps in $H^{d}$.

3. Blaschke type perturbation. Let $U \subset B(f)$ be an open topological disc containing $p$, with smooth boundary not containing critical points, such that $f(\operatorname{cl} U) \subset U, f: U \rightarrow f(U)$ is a proper map and $d^{\prime}:=\operatorname{deg}\left(\left.f\right|_{U}\right) \geq 2$. Then we construct a 1-parameter family of maps joining in $H(f)$ the map $f$ to a map having $p$ as a $d^{\prime}$-multiple fixed point as follows:

Let $R_{1}, R_{2}$ be Riemann maps from $U$, resp. $f(U)$, to the unit disc $\mathbb{D}$ such that $R_{i}(p)=0, i=1,2$. Let $a_{1}=0, a_{2}, \ldots, a_{d^{\prime}}$ be the $R_{1}$-images of the $\left.f\right|_{U}$-preimages of $p$. Let

$$
B_{t}=\lambda z \prod_{i=2}^{d^{\prime}} \frac{z-t a_{i}}{1-t \bar{a}_{i} z}, \quad|\lambda|=1, t \in[0,1] .
$$

We set $h_{t}=R_{2}^{-1} \circ B_{t} \circ R_{1}$. Here $\lambda$ is chosen so that $h_{1}=f$. It is useful to write $h_{t}=R_{1}^{-1} \circ g_{t} \circ R_{1}$, where $g_{t}=R_{1} \circ R_{2}^{-1} \circ B_{t}$. Change $h_{t}$ in $U$ close to $\partial U$ by a smooth isotopy so that $h_{t}$ and $f$ coincide on $\partial U$ for all $t$. We extend $h_{t}$ outside $U$ by $f$ to the whole $\overline{\mathbb{C}}$. As in the previous cases we pull back the standard conformal structure from $R_{1}(f(U))$ to $f(U)$ by $R_{1}^{-1}$, 
extend it by $h_{t}^{-n}$, complete on $\overline{\mathbb{C}} \backslash \bigcup h_{t}^{-n}(f(U))$ with the standard structure and integrate.

Let $\Phi_{f}$ conjugate $f$ to $z \mapsto \lambda z$, where $\lambda:=f^{\prime}(p) \neq 0$, in a neighbourhood of $p$ (i.e. $\left.\Phi_{f}(f(z))=\lambda \Phi_{f}(z)\right)$. Extend $\Phi_{f}$ to $B(f)$ by $\Phi_{f}(z)=$ $\lambda^{-n} \Phi_{f}\left(f^{n}(z)\right)$. Define $G_{f}(z)=\left|\Phi_{f}(z)\right|^{2}$.

If $f \in H^{d}$ and $g \in H(f)$ then we write $p_{g}$ for the point $z$ with $g(z)=z$ such that $(g, z)$ belongs to the component of the Cartesian product $H(f) \times \overline{\mathbb{C}}$ containing $\left(f, p_{f}\right)$. There exist $B(g)$ and $\Phi_{g}$ (provided $\left.g^{\prime}\left(p_{g}\right) \neq 0\right)$ as above.

Now we can formulate

Main Lemma. For every $f \in H^{d}$ there exists $g \in H(f)$ such that $g^{\prime}\left(p_{g}\right) \neq 0$ and there exists $a>0$ such that all critical values for critical points in $B(g)$ are in a component $\partial$ of $\left\{G_{g}=a\right\}$ which is a topological circle separating $p_{g}$ from the Julia set $J(g)$.

Proof. By small perturbations (types 2 and 3) we assure that the sink $p_{f}$ is neither a critical point nor a critical value for a critical point in $B=B(f)$, all critical points in $B$ are simple and their forward trajectories are pairwise disjoint.

At the end it may occur useful also to have $\frac{1}{\pi} \operatorname{Arg} f^{\prime}(p)$ irrational. We assure this by a type 3 perturbation where $\operatorname{Arg} \lambda$ is the parameter.

The critical points of $G=G_{f}$ are

1) the fixed point $p$ and its iterated pre-images (these are minimum points with $G=0$ ),

2) the critical points of $f$ and their iterated pre-images (these are saddle points for $G$ ).

Denote the set of points in 1) by $M$ and the set of points in 2) by $S$.

For every $q \in M$ let $A(q)$ denote the basin of attraction to $q$ for the flow of the vector field $-\operatorname{grad} G$. (Note that the boundary of $A(q)$ is the union of a set of some points from $S$ and their stable separatrices.)

Denote by $r(q)$ the least non-negative integer such that $f^{r(q)}(q)=p$. For every $z \in A(q)$ or $X \subset A(q)$ write $r(z):=r(q)$ and $r(X):=r(q)$.

Observe that for every $z \in B(f)$ there exists a curve $\gamma$ joining $z$ to $p$ consisting of critical points of $G$ and of trajectories of $\operatorname{grad} G$ where this field is non-zero (i.e. $\gamma$ goes from $z$ to a critical point, say a minimum, then to a saddle, then to a minimum etc. until it reaches $p)$. Let $\gamma(z)$ denote a curve as above intersecting the least possible number of $A(q)$ 's. Denote the number of these $A(q)$ 's by $s(z)$ or $s_{f}(z)$ and call the curve a Morse curve.

OBSERVATIONS. 1. $r(f(z))=r(z)-1$ if $r(z) \geq 1$.

2. $s(f(z)) \leq s(z)$. 
Observation 2 follows from the fact that $f$ maps trajectories of $\operatorname{grad} G$ to trajectories of $\operatorname{grad} G$.

The plan is now to move all critical values to the same level $G=a$ in $A(p)$.

We shall do this for each critical point separately so that we do not move the critical values moved before to level $a$ in $A(p)$. We move each critical value $f(c)$ step by step so that after each step, $s(f(c))$ decreases and $f(c)$ is in some $A(q)$. When $f(c) \in A(p)$ we move $f(c)$ along the trajectory of $\operatorname{grad} G$ to level $a$ as described in 1 .

Take $c \in B(f)$, a critical point for $f$. By a small perturbation in a neighbourhood of $c$ we obtain $f(c) \in A(q)$. We can assume that $q \neq p$. This is correct because:

(a) The perturbation is above the $G$-level of $f(c)$ so it does not change the part of the stable manifolds of a saddle to which $f(c)$ might have belonged, in a neighbourhood of $f(c)$ and between it and the saddle.

(b) The change of coordinates integrating the new invariant conformal structure is conformal there. This is so because the structure is non-standard only in some places above $f(c)$. Thus the change of coordinates maps the gradient lines of the old $G$ to the gradient lines of the new one and stable manifolds (separatrices) to separatrices.

Now let $\gamma$ be the part of a Morse curve $\gamma(f(c))$ joining $f(c)$ to the first saddle $\omega \in \gamma(f(c))$.

Observe that for every $j>0$ we have $f^{j}(\gamma) \cap \gamma=\emptyset$.

Indeed, we have $f^{j}(\gamma \cap A(q)) \cap \gamma=\emptyset$ by Observation 1. By the same observation, $f^{j}(\omega) \in \operatorname{cl} A\left(f^{j}(q)\right)$ cannot be in $\gamma \cap A(q)$. Finally, $f^{j}(\omega) \rightarrow p$ so $f^{j}(\omega) \neq \omega$.

As $f^{j}(\gamma) \rightarrow p$ we can find a neighbourhood $U$ of $\gamma$ so that $f^{j}(U) \cap U=\emptyset$ for all $j>0$. Take in $U$ a curve $\gamma^{\prime}$ joining $f(c)$ to a point $z$ close to $\omega$ with $z \in A\left(q^{\prime}\right)$ and $s\left(q^{\prime}\right)=s(q)-1$. Take care to choose $\gamma^{\prime}$ disjoint from $V=$ the set of all critical values under iterates of $f$ except $f(c)$.

Now perturb $f$ along $\gamma^{\prime}$ as described in 1. Do it with $g_{t}$ different from identity only in a neighbourhood of $\gamma^{\prime}$ small enough to be disjoint from $V$. The condition (1) is of course satisfied.

Now let us explain why the new map $f_{1}$ has the property

$$
s_{f_{1}}\left(f_{1}\left(c_{f_{1}}\right)\right)=s_{f}(f(c))-1 .
$$

Here $c_{f_{1}}$ is the old $c$ in the new coordinates; it is a critical point for $f_{1}$. We use the fact that the part of the domain where we changed $f$ to $h_{t}$ is in the basins $A\left(f^{-1}(q)\right)$, where by Observation $2, s \geq s(q)$. So we did not change $G$ in the basins and the part of the Morse curve $\gamma(f(c))$ beyond 
$\omega$ goes through, where $s<s(q)$. We also changed $f$ in a neighbourhood of $f^{-1}(\omega)$. This does not change $G$ below a neighbourhood of $\omega$ because $G\left(f^{-j}(\omega)\right)>G(\omega), j=1,2, \ldots$ (Compare this with the argument (a).) This does not change $G$ below the part of $\gamma(f(c))$ beyond a neighbourhood of $\omega$ either, because this change is close to the set $s \geq s(q)$.

The change of $h_{t}$ to $f_{t}$ does not affect (2). Indeed, also by the above arguments the new measurable conformal structure is the standard one below $\omega$ and the part of $\gamma(f(c))$ beyond $\omega$. So the change of coordinates maps the gradient lines of the old $G$ to the gradient lines of the new one as in (b).

When $f(c) \in A(p)$ we first make a small perturbation so that the gradient line of $G$ passing through $f(c)$ does not intersect the forward trajectories of other critical values which are already in $A(p)$. Next we move $f(c)$ along $\gamma$ which is a piece of the gradient line of $G$ passing through $f(c)$ joining it to $\{G=a\}$ in $A(p)$. We succeed because $\frac{1}{\pi} \operatorname{Arg}\left(f^{n}\right)^{\prime}(p)$ is irrational so all the curves $f^{n}(\gamma), n \geq 0$, are pairwise disjoint and the conformal structure does not change below these curves.

After a sequence of consecutive perturbations as above we obtain a rational mapping $g$ with all the critical values on one level $\{G=a\}$, more precisely, on its component $\partial$ intersecting $A(p)$.

Otherwise cl $D_{a}$ contains an $S$-type critical point $x$ for $G_{g}$. Then there exists $n>0$ such that $y=g^{n}(x)$ is a critical value for $g$. Hence $G_{g}(y)=$ $\left|g^{\prime}\left(p_{g}\right)\right|^{n} G_{g}(x)<G_{g}(x) \leq a$. This contradicts $G_{g}(y)=a$ just achieved.

The proof of the Main Lemma is finished.

Proof of Theorem A. Let $f$ be already as $g$ in the Main Lemma. By perturbing along curves one obtains additionally all critical values for critical points from $f^{-1}(B(f)) \backslash B(f)$ also in $\partial$. (A posteriori we will see that under the assumptions of Theorem A we have $f^{-1}(B(f)) \backslash B(f)=\emptyset$, i.e. $B(f)$ is completely invariant.)

Denote again the domain of $\overline{\mathbb{C}} \backslash \frac{\partial}{C}$ containing $p$ by $D_{a}$. Denote the complementary open topological disc $\overline{\mathbb{C}} \backslash \operatorname{cl} D_{a}$ by $D_{a}^{\prime}$.

Observations. 3. There is at most one critical value for $f$ in $D_{a}^{\prime}$. So the components of $f^{-1}\left(D_{a}^{\prime}\right)$ are topological discs $D^{\prime j}, j=1, \ldots, \widehat{d}$ where $\widehat{d} \leq d$, with closures in $D_{a}^{\prime}$. (In particular, $f^{-1}\left(\mathrm{cl} D_{a}\right)$ is connected and hence $B(f)$ is completely invariant.)

4. The closures of $D^{\prime j}$ intersect each other or "self-intersect" only at critical points of $f$ and $\mathrm{cl} f^{-1}\left(D_{a}^{\prime}\right)$ is connected (see Fig. 1).

If the latter were false then $f^{-1}\left(D_{a}\right)$ would contain a non-simply connected component $V$. But $f$ maps $V$ onto the disc $D_{a}$ so $V$ would contain a critical point, hence $D_{a}$ would contain a critical value. This would contradict the assumption that all critical values are on level $a$. 
After a small perturbation moving (exposing) critical values of some $d-1$ critical points towards below $a$, the set $\operatorname{cl} f^{-1}\left(D_{a}^{\prime}\right)$ consists of $\widehat{d}$ closed discs intersecting one another in at most 1 point, whose union is connected and simply connected (Fig. 2).

So for $\varepsilon>0$ small enough the set $f^{-1}\left(\partial D_{a-\varepsilon}\right)$ (where $D_{a-\varepsilon}:=D_{a} \cap\{G<$ $a-\varepsilon\})$ is a topological circle $\partial_{\varepsilon}$ bounding a topological disc $U \ni p$ and under $f$ it winds $d$ times onto $\partial D_{a-\varepsilon}$. Of course $f(\operatorname{cl} U)=\operatorname{cl} D_{a-\varepsilon} \subset U$.

After performing a Blaschke type perturbation and a holomorphic change of coordinates on $\overline{\mathbb{C}}$ we arrive at a polynomial.

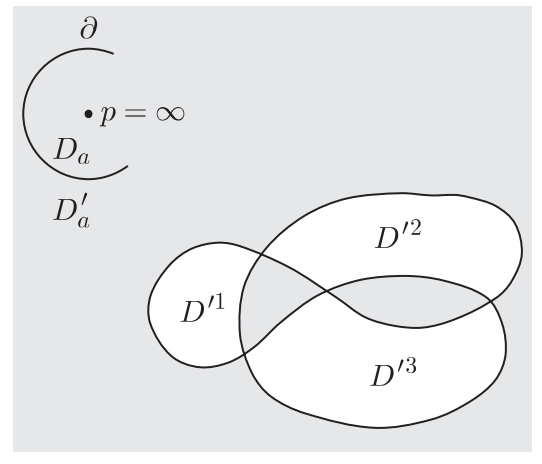

Fig. 1

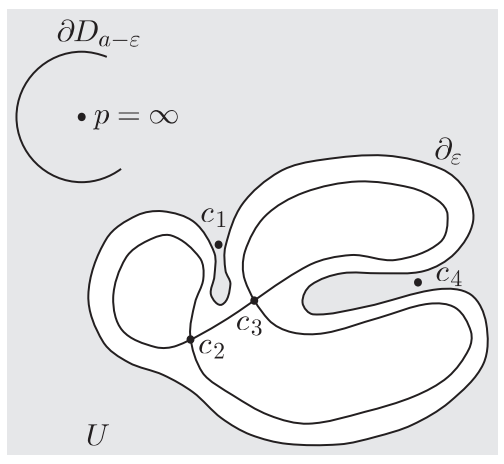

Fig 2. Critical points $c_{1}$ and $c_{4}$ are exposed towards $p$

Proof of Corollary B. This corollary follows from Theorem A because its assertion is an open property in $H^{d}$ and it is true and easy for polynomials.

Indeed, if $f$ is a polynomial, then with the help of a small perturbation we guarantee that for each critical value $v_{j}, j=1, \ldots, d-1$ (different from $\infty)$, the trajectory $\gamma_{j}$ for $\operatorname{grad} G$ where $G$ is Green's function in the basin of attraction to $\infty$, with pole at $\infty$, goes from $v_{j}$ up to $\infty$. In other words, $\gamma_{j}$ does not go to any critical point for $G$. Then there are no critical values for $f$ in the topological disc

$$
U=\overline{\mathbb{C}} \backslash A, \quad \text { where } \quad A=\bigcup_{j=1, \ldots, d-1, n \geq 0} f^{n}\left(\gamma_{j}\right)
$$

Since $f(A) \subset A$, we have a collection of branches $g_{j}: U \rightarrow U$ of $f^{-1}$. Denote $g_{j}(U)$ by $U_{j}$. Then each $z \in J(f)$ is coded by the sequence of symbols $j_{n}$, $n=0,1, \ldots$, where $f^{n}(z) \in U_{j_{n}}$.

For each sequence $\left(j_{n}\right)$ the family of maps $\Psi_{n}=g_{j_{0}} \circ g_{j_{1}} \circ \ldots \circ g_{j_{n}}$ is a normal family of maps on $U$. It is easy to find a slightly smaller topological 
$\operatorname{disc} U^{\prime} \subset U$ so that $U^{\prime} \supset J(f)$ and

$$
\text { each } g_{j} \text { maps cl } U^{\prime} \text { into } U^{\prime} \text {. }
$$

Hence the sequences $\left.\Psi_{n}\right|_{U^{\prime}}$ converge uniformly to points in $J(f)$. This proves that the coding is one-to-one.

R e m a r k 1.1. In fact, a proof of Corollary B is contained in the proof of Theorem A. Indeed, already Observation 3 gives a partition of the Julia set $J$ into $J \cap D^{\prime j}$, and $J \ni z \mapsto\left(j_{n}\right)$ such that $f^{n}(z) \in D^{\prime j_{n}}$ gives a conjugacy to the one-sided shift on the space of $d$ symbols.

Observe that for this proof of Corollary B, there was no need to refer to the Measurable Riemann Mapping Theorem. Namely, there was no need to integrate every time a new conformal structure to obtain a rational mapping. One could just work with smooth maps until the properties described in Observation 3 were reached.

Remark 1.2. One can prove Theorem A by perturbing $f$ along a curve $\widehat{\gamma}$ close to the Morse curve, dragging the critical value to a small neighbourhood of $p$ in just one step instead of doing it step by step decreasing $s(f(c))$. The property (1) is satisfied by Observations 1 and 2. (Every point of $\widehat{\gamma}$ can come close to $\widehat{\gamma}$ under $f^{j}$ only further, i.e. if $f^{j}(\widehat{\gamma}(\tau))$ is close to $\widehat{\gamma}\left(\tau^{\prime}\right)$ then $\tau^{\prime}>\tau$. The more so for $f^{j}$ replaced by $h_{t}^{j}$.)

Remark 1.3. Makienko $[\mathrm{M}]$ proved the following Proposition which corresponds to our Main Lemma:

If all critical values $v_{1}, \ldots, v_{m}$ for critical points in the basin $B(f)$ of immediate attraction to an attracting fixed point $p_{f}$ have disjoint forward orbits then there exists a topological disc $U \subset B(f)$ containing $p_{f}$ such that $\left.f\right|_{U}$ is injective, $f(\mathrm{cl} U) \subset U$ and all $v_{j}, j=1, \ldots, m$, belong to the annulus $U \backslash f(U)$.

One can easily change $U$ so that all $v_{j}$ belong to $\partial f(U)$. There is a quasiconformal conjugacy $\Phi$ of $f$ on $U$ to $z \mapsto \lambda z$ on the unit disc $\mathbb{D},|\lambda|<1$. So as usual one can pull back the standard conformal structure on $\mathbb{D}$ by $\Phi^{-1}$ to $U$, spread it by $\Phi^{-n}, n=1,2, \ldots$, to the whole basin of $\partial_{f}$ and complete on the complement of the basin by the standard structure. After integration of this structure the new map satisfies the properties asserted in the Main Lemma.

Thus: Makienko's Proposition (preceded by a small perturbation) + the Measurable Riemann Mapping Theorem give the Main Lemma.

Conversely, $g$ from the assertion of the Main Lemma is conjugate to $f$ (provided $f$ is already after the first perturbation in the proof of the Main Lemma). So Makienko's $f(U)$ can be defined as the image under the conjugacy of the disc bounded by $\partial$. Observe that for that we did not need 
to construct new holomorphic structures along the proof. (Compare this with Remark 1.1.)

Thus Makienko's Proposition is the topological heart of the Main Lemma.

Remark 1.4. Neither in the Main Lemma nor in Theorem A does one need to assume $f \in H^{d}$. The proofs work for an arbitrary rational map $f$ with $\operatorname{deg} f \geq 2$ if one replaces in the statements $H(f)$ by Teich $J$. The latter denotes the set of all rational maps $g$ such that there exists

1) a connected open domain $\mathcal{P} \ni 0$ in the complex plane,

2) a family of quasiconformal homeomorphisms $h_{\lambda}: \overline{\mathbb{C}} \rightarrow \overline{\mathbb{C}}, \lambda \in \mathcal{P}$, with $h_{0}=\mathrm{id}$ such that for each $z$ the point $h_{\lambda}(z)$ depends holomorphically on $z$ (such a family is usually called a holomorphic motion),

3) a family of rational maps $f_{\lambda}, \lambda \in \mathcal{P}$, with $f_{0}=f$ and $f_{\lambda_{0}}=g$ for some $\lambda_{0} \in \mathcal{P}$ such that for every $\lambda$ the map $h_{\lambda}$ conjugates $f$ with $f_{\lambda}$ between $\varepsilon$-neighbourhoods of their Julia sets ( $\varepsilon$ not depending on $\lambda$ ).

Proof of Theorem C. One first proves an analogue to the Main Lemma:

If $f(z)=z+a(z-p)^{t+1}+o\left((z-p)^{t+1}\right)$ with $a \neq 0$ then $H_{1}(z)=\lambda / z^{t}$ for some $\lambda \neq 0$ conjugates $f$ on a "petal" $P \subset B(f)$ to $F(z)=z+1+o(1)$ for $z$ with large real part. (See [DH2] for the precise description.) Next conjugate $F$ smoothly to $z \mapsto z+1$ by $H_{2}$. Define $\Phi_{f}=H_{2} \circ H_{1}$ on $P$ and extend it to $B(f)$ by $\lim _{n \rightarrow \infty}\left(\Phi_{f} \circ f^{n}(z)-n\right)$. Define $G_{f}(z):=1 / \exp \Re \Phi_{f}(z)$ on $B(f)$.

Then after a small perturbation such that all critical points in $B$ are simple and their forward orbits are disjoint, one can find a quasiconformally conjugate $g \in Q^{d}$ such that all critical values $v_{j}$ for $g$-critical points in $B(g)$ are in the component of $\left\{G_{g}=a\right\}$ bounding a "petal". (See Fig. 3.)

The proof of this parabolic version of the Main Lemma is the same as that of the Main Lemma except that there are no $M$-critical points for $G_{f}$ in $B(f)$. One can think of $M$-critical points as belonging to $\partial B(f)$, forming precisely the set $\partial B(f) \cap \bigcup_{n \geq 0} f^{-n}(\{p\})$. So the perturbation is not along Morse curves in basins $A(q)$ but along curves in $B(f) \cap A(q)$ joining directly consecutive $S$-critical points in $\partial A(q)$.

Repeat now the proof of Corollary B:

(The assumptions imply $t=1$.)

For $f$ already as $g$ above we take curves $\gamma_{j}, j=1, \ldots, 2 d-2$, joining $v_{j}$ to $p$. We can take as $\gamma_{j}$ 's the $\Phi_{f}^{-1}$-preimages of horizontal lines in $\overline{\mathbb{C}}$. Then $f\left(\gamma_{j}\right) \subset \gamma_{j}$. So for $A=\bigcup_{j=1}^{d-1} \gamma_{j}$ the set $\overline{\mathbb{C}} \backslash A$ is a topological disc and for the branches $g_{j}$ of $f^{-1}$ we have $g_{j}(U) \subset U, j=1, \ldots, d$.

We find $U^{\prime} \subset U$ such that (3) holds, except that $\partial U^{\prime} \cap \partial U=\{p\}$ and $g_{j_{0}}(p)=p$ for the branch $g_{j_{0}}$ with $g_{j_{0}}(p)=p$ (see Fig. 3 ). Since $\left(g_{j_{0}}^{n}\right)^{\prime} \rightarrow 0$ 


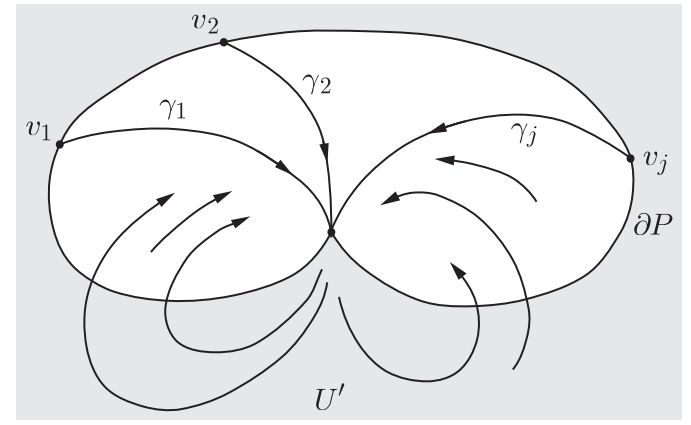

Fig. 3

on $U^{\prime}$ we conclude that $\bigcap_{n} g_{j_{0}}^{n}\left(\operatorname{cl} U^{\prime}\right)$ is only one point $p$.

Re m a r k 1.5. As mentioned in the introduction, the fact that under the assumptions of Corollary B or Theorem $\mathrm{C}, J(f)$ is a Cantor set, is much easier than the fact that $\left.f\right|_{J(f)}$ is conjugate to the one-sided shift.

Indeed, it is easy to prove that $\left.f^{N}\right|_{J(f)}$ is conjugate to a one-sided shift, for an integer $N>0$. Just take a small topological disc $D \subset B(f)$ so that $D \ni p$ (or a small petal in the parabolic case) such that $f(\operatorname{cl} D) \subset D$. For each critical value $v_{j}$ and every $n \geq 0$ such that $f^{n}\left(v_{j}\right) \notin D$ join $f^{n}\left(v_{j}\right)$ to $D$ by an embedded curve $\gamma_{j, n}$ so that these curves are mutually disjoint. Take $N$ such that $f^{N}\left(\gamma_{j, n}\right) \subset D$ for each $j, n$. Consider now $U=\overline{\mathbb{C}} \backslash A$, where $A=D \cup \bigcup_{j, n} \gamma_{j, n}$. Next proceed as in the proof of Corollary B with $f$ replaced by $f^{N}\left({ }^{2}\right)$.

Remark 1.6. It is also easy to prove that $\left.f\right|_{J(f)}$ is conjugate to a one-sided topological Markov chain.

Indeed, let $D_{0}=D$ as in Remark 1.5. Let $D_{n}, n=1,2, \ldots$, be defined recursively: $D_{n}$ is the component of $f^{-1}\left(D_{n-1}\right)$ containing $D_{n-1}$. Let $N \geq 0$ be large enough that there are no critical values in $\overline{\mathbb{C}} \backslash D_{N}$. Let $U_{1}, \ldots, U_{K}$ denote all the components of $\overline{\mathbb{C}} \backslash \operatorname{cl} D_{N}$. These are topological discs because $D_{N}$ is connected. Consider the family of topological discs $g_{j}\left(U_{k}\right)$ for all branches $g_{j}, j=1, \ldots, d$, of $f^{-1}$ and $k=1, \ldots, K$. Then for every sequence of pairs $\left(j_{n}, k_{n}\right), n=0,1, \ldots$, such that $g_{j_{n}}\left(U_{k_{n}}\right) \subset U_{k_{n+1}}$ there exists precisely one point $z \in J(f)$ such that $f^{n}(z) \in g_{j_{n}}\left(U_{k_{n}}\right)$ for every $n \geq 0$.

R e mark $1.7\left({ }^{3}\right)$. Considering the situation in Theorem A such that $p$ is already not critical (say all $f$-critical points belong to $B(f)$ and $\operatorname{Arg} 2 \pi f^{\prime}(p)$ is rational), John Milnor asked whether it is possible to find a set $A$ which is the union of parts of trajectories of $\operatorname{grad} G$, contains all critical values, is

\footnotetext{
$\left({ }^{2}\right)$ I owe this proof to K. Barański.

$\left({ }^{3}\right)$ Before reading this remark the reader is advised to read Section 2.
} 
compact in $B(f), f$-forward invariant, connected and simply connected (a tree).

This would immediately allow us to prove Corollary B with the set $A$ as here.

Unfortunately, the answer is negative. We can modify an exotic example of Section 2 (the case $d=3$, Fig. 5) so that $f\left(c_{4}\right)=f^{2}\left(c_{3}\right)=a$, the pole. Then the set of saddles for $G$ is $S=\bigcup_{n>0} f^{-n}\left(c_{2}\right)$. So every curve $\Gamma$ built from pieces of trajectories of $\operatorname{grad} G$ joining $f\left(c_{4}\right)$ to $\infty$ passes through a point of $f^{-n}\left(\left\{c_{2}\right\}\right)$ for some $n$ (in fact, $n>0$ ). Hence $f^{n}(\Gamma)$ joins $\infty$ to $\infty$ passing through $c_{2}$, hence it is a loop, i.e. $A$ is not a tree.

(Even the assumption $f\left(c_{4}\right) \neq a \neq f^{2}\left(c_{3}\right)$ does not help if $f\left(c_{4}\right), f^{2}\left(c_{3}\right)$ are close to $a$. $\Gamma$ must still leave the basin $A(a)$ for $\operatorname{grad} G$, passing through a point in $\bigcup_{n \geq 0} f^{-n}\left(\left\{c_{2}\right\}\right)$, and for typical $f\left(c_{4}\right)$, first meets the point $a$.)

Remark $1.8\left(^{3}\right)$. Though in the Main Lemma we can arrange to have all critical values for critical points in $B(f)$ on one component of a level of $G$, it is sometimes not so for critical points (unless all critical points for $f$, or all but one, are in $B(f)$ as in Theorem A, see Fig. 2). Again we modify an exotic example of Section 2. Here the degree of $f$ is 5. Start with a cubic polynomial $P$ which has degree 1 on $\partial A_{1}$ and degree 2 on $\partial A_{2}$ (see Fig. 3, Section 2), and $P$ maps the critical point $a \in A_{2}$ to the critical point at the self-intersection of the figure 8 line $\partial A_{1} \cup \partial A_{2}$, which escapes to $\infty$.

Consider now the function $z \mapsto P(z)+b /(z-a)^{2}$ with $b$ a small real positive number. We obtain the picture of Figure 4 (do the surgery as in Section 2 to find appropriate $a$ and $b$ ). For a final example split $a$ into two different poles, which gives an $f$-critical point $c_{8}$ between them (Fig. 4). Make a Blaschke type perturbation close to $\infty$ to have $\infty$ not critical and move $f\left(c_{8}\right), f\left(c_{1}\right), f\left(c_{2}\right)$ to one level with $f\left(c_{3}\right)$.

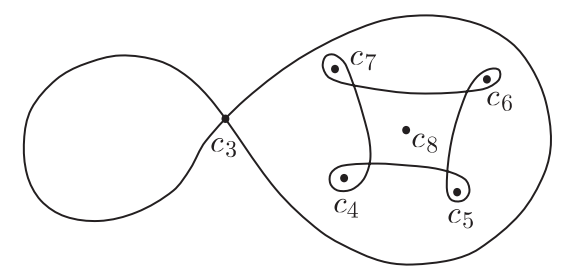

Fig. 4. $f^{2}\left(c_{4}\right)=c_{4}, f^{2}\left(c_{6}\right)=c_{6}, f\left(c_{5}\right)=c_{5}, f\left(c_{7}\right)=c_{7}$

\section{Exotic basins}

Proof of Theorem D, case $d=3$. We start with the geometric description of an exotic example of degree 3, illustrated in Fig. 5.

Start with a quadratic polynomial $P(z)=z^{2}+c$ with the critical point $c_{2}=0$ escaping to $c_{1}=\infty$, which is an attracting fixed point of multipli- 
city 2. The level $\partial=\{G=t\}$ of Green's function of the basin of attraction to $\infty$ with pole at $\infty$, containing $c_{2}$, is a figure 8 . Now we change the map on $B_{2}$, one of the two discs $B_{1}, B_{2}$ bounded by $\partial$, as follows:

Draw two little discs $D_{1}, D_{2}$ in $B_{2}$, intersecting each other. Let $D_{1} \cap D_{2}$ be mapped 1-to-1 onto $\overline{\mathbb{C}} \backslash\left(B_{1} \cup B_{2}\right)$. Let $D_{1} \backslash D_{2}$ go onto $B_{1}$ and $D_{2} \backslash D_{1}$ go onto $B_{2}$, both via proper maps with degree 2 . So there are critical points $c_{3} \in D_{1} \backslash D_{2}$ and $c_{4} \in D_{2} \backslash D_{1}$. On $D_{2}$ this map $f$ is quadratic-like so we can do anything there, for example $f\left(c_{4}\right)=c_{4}$. On $D_{1}$ the map $f^{2}$ is quadratic-like so we can assume $f^{2}\left(c_{3}\right)=c_{3}$.

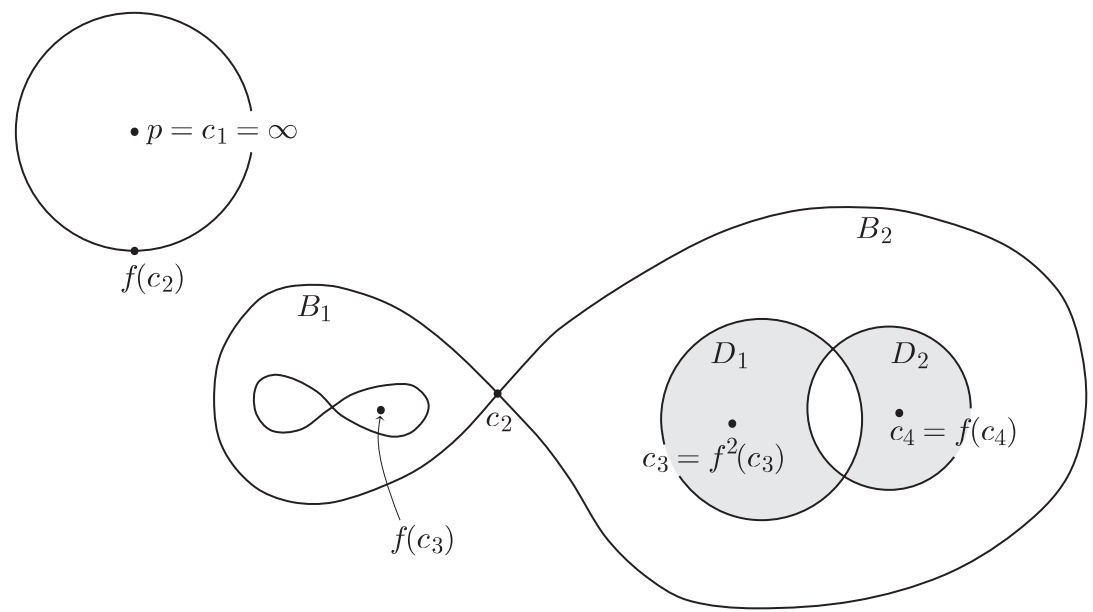

Fig. 5

The required rational function is obtained from this topological picture by the quasiconformal surgery technique $[D]$. We now explain it in more detail.

We need the following lemma which generalizes Douady-Hubbard's theorem that a polynomial-like mapping is quasiconformally conjugate to a polynomial [DH1]:

LEMMA 2.1. Let $U \subset \overline{\mathbb{C}}$ be an open set (not necessarily connected or simply connected) with boundary being a union of smooth Jordan curves. Let $F_{1}: U \rightarrow U$ be a holomorphic map such that $F_{1}(\mathrm{cl} U) \subset U$. (We denote the continuous extension of $F_{1}$ to $\mathrm{cl} U$ by the same symbol.) Let $V \subset \overline{\mathbb{C}}$ be homeomorphic to $\overline{\mathbb{C}} \backslash \mathrm{cl} U$ by a homeomorphism $h_{1}$ which extends to an orientation preserving homeomorphism of $\overline{\mathbb{C}}$. (Again we do not assume $V$ is connected or simply connected.) Let $F_{2}: V \rightarrow \overline{\mathbb{C}}$ be a holomorphic map. Also, suppose that the boundary of $V$ is smooth and denote by $F_{2}$ the continuous extension of the original $F_{2}$ to $\mathrm{cl} V$. Suppose the family of curves consisting of the components of $\partial U$ and their $F_{1}$-images has the same 
combinatorics in $\overline{\mathbb{C}}$ as the family of curves consisting of the components of $\partial \mathrm{V}$ and their $F_{2}$-images, in the sense that

1. There exists a standard orientation preserving homeomorphism $h_{2}$ : $\partial U \cup F_{1}(\partial U) \rightarrow \partial V \cup F_{2}(\partial V)$ such that the boundary of each component $K$ of $\overline{\mathbb{C}} \backslash\left(\partial U \cup F_{1}(\partial U)\right)$ is mapped to the boundary of a component of $\overline{\mathbb{C}} \backslash(\partial V \cup$ $\left.F_{2}(\partial V)\right)$ and the map extends $Q$-quasiconformally on $K$ with a constant $Q$ independent on $K$.

2. For each component $\partial$ of $\partial U$ the map $h_{2}$ maps $\partial$ to $h_{1}(\partial), F_{1}(\partial)$ to $F_{2}\left(h_{1}(\partial)\right)$ and moreover there is a homeomorphism (lift) $\widetilde{h}_{2}: \partial \rightarrow h_{1}(\partial)$ such that on $\partial$ we have $h_{2} \circ F_{1}=F_{2} \circ \widetilde{h}_{2}$.

Then there exists a rational map $f: \overline{\mathbb{C}} \rightarrow \overline{\mathbb{C}}$ and an open set $W \subset \overline{\mathbb{C}}$ such that $f$ is quasiconformally conjugate to $F_{1}$ on $W$ and quasiconformally conjugate to $F_{2}$ on $\overline{\mathbb{C}} \backslash W$.

Note that the curves $\partial \rightarrow F_{1}(\partial)$ can intersect each other or self-intersect. In particular, $\partial$ can map to a Jordan curve with degree larger than 1.

Proof. We replace $h_{2}$ on $\partial U$ by the lift $\widetilde{h}_{2}$ and then extend it from $\partial U \cup F_{1}(\partial U)$ to a quasiconformal homeomorphism $h: \overline{\mathbb{C}} \rightarrow \overline{\mathbb{C}}$. Define $F: \overline{\mathbb{C}} \rightarrow \overline{\mathbb{C}}$ to be $F_{2}$ on $\mathrm{cl} V$ and $h \circ F_{1} \circ h^{-1}$ on $\overline{\mathbb{C}} \backslash V$.

Let $\mu_{0}$ denote the standard conformal structure on $\overline{\mathbb{C}}$. Take $\mu_{1}=$ $h_{*}\left(\left.\mu_{0}\right|_{U}\right)$ on $\overline{\mathbb{C}} \backslash \mathrm{cl} V$. (Think about $\mu_{1}$ as a field of ellipses, up to multiplication by a positive function.) For each $z \in V$ define $\mu_{1}(z)$ as a pull-back $F_{*}^{-n}\left(\mu_{1}\right)\left(F^{n}(z)\right)$, where $n \geq 0$ is such that $F^{n}(z) \in \overline{\mathbb{C}} \backslash \mathrm{cl} V$. If no such $n$ exists, take $\mu_{1}(z)=\mu_{0}(z)$. This is correct due to the crucial property $F(\overline{\mathbb{C}} \backslash \operatorname{cl} V) \subset \overline{\mathbb{C}} \backslash \operatorname{cl} V$. As $F_{2}$ is holomorphic, $\mu_{1}$ is in $L^{\infty}$ !

Now integrate $\mu_{1}$. In the new coordinates $F$ changes to the rational map $f$ we looked for.

Now we construct $F_{1}$ and $F_{2}$ satisfying the assumptions of Lemma 2.1. This is illustrated in Fig. 6.

Take the polynomial $P(z)=z^{2}+c, c<-2$. Define $F_{1}$ by adding to $P$ a term $b /(z-a)$ for $a=\sqrt{-c} \in P^{-1}(0) \cap B_{2}(a>0)$. Let $b>0$ be so small that for our $F_{1}$ the level $\widehat{\partial}=\left\{\widehat{G}=t_{0}\right\}$ containing the $F_{1}$-critical point $\widehat{c}_{2}$ close to $c_{2}=0$ is a figure 8 close to $\partial$. Here $\widehat{G}$ is defined analogously to Green's function or to $G$ in Section 1: on the basin of attraction to $\infty$ by $F_{1}$, one defines $\widehat{G}(z)=\lim _{n \rightarrow \infty} 2^{-n} \log \left|F_{1}^{n}(z)\right|$. Denote discs bounded by appropriate parts of $\widehat{\partial}$ close to $B_{1}, B_{2}$ by $\widehat{B}_{1}, \widehat{B}_{2}$ respectively.

It is easy to compute that $\widehat{c}_{2}=b /\left(2 a^{2}\right)+o(b)$ and for the two other $F_{1}$ critical points $\widehat{c}_{3}, \widehat{c}_{4}$ we have $F_{1}\left(\widehat{c}_{3,4}\right)=\mp 2 \sqrt{2 a} \sqrt{b}+o(\sqrt{b})$. So $F_{1}\left(\widehat{c}_{3}\right) \in \widehat{B}_{1}$ and $F_{1}\left(\widehat{c}_{4}\right) \in \widehat{B}_{2}$. 
Let $0<t_{2}<t_{1}<t_{0}$ with $t_{2} \approx t_{1} \approx t_{0}$ and denote by $K_{2}, K_{1}$ the topological discs both in $\widehat{B}_{1}$ bounded by $\left\{\widehat{G}=t_{2}\right\}$, resp. $\left\{\widehat{G}=t_{1}\right\}$. Denote by $K_{4}^{\prime}$ the topological disc in $\widehat{B}_{2}$ bounded by $\left\{\widehat{G}=t_{1}\right\}$. Finally, denote the component of $F_{1}^{-1}\left(K_{1}\right)$ in $\widehat{B}_{2}$ by $K_{3}$, and the component of $F_{1}^{-1}\left(K_{4}^{\prime}\right)$ in $\widehat{B}_{2}$ by $K_{4}$. We have $\widehat{c}_{3} \in K_{3}$ and $\widehat{c}_{4} \in K_{4}$.

Define $U:=\overline{\mathbb{C}} \backslash\left(K_{2} \cup K_{3} \cup K_{4}\right)$. We have $F_{1}(\operatorname{cl} U) \subset U$. So $F_{1}$ and $U$ satisfy the assumptions of Lemma 2.1. Now we need to define $F_{2}$ :

Set $F_{2}(z):=z^{2}$ on a geometric disc $L_{4}=\left\{|z|<r_{4}\right\}, r_{4}>1$. Take a disc $L_{3}=\left\{\left|z-z_{0}\right|<r_{3}\right\} \subset F_{2}\left(L_{4}\right) \backslash \operatorname{cl} L_{4}$ and define $\widetilde{F}_{2}(z)=\left(z-z_{0}\right)^{2}+z_{0}$. One finds large $r_{4}, r_{3}$ such that $\widetilde{F}_{2}\left(L_{3}\right) \supset \mathrm{cl} F_{2}\left(L_{4}\right)$. Pick in $\widetilde{F}_{2}\left(L_{3}\right) \backslash F_{2}\left(L_{4}\right)$ two $\operatorname{discs} L_{2} \subset L_{1}$ of the form $L_{1}=\left\{\left|z-z_{1}\right|<r_{1}\right\}$ and $L_{2}=\left\{\left|z-z_{1}\right|<r_{2}\right\}$ with $r_{2}<r_{1}$. Take an affine holomorphic map $\Psi: L_{1} \rightarrow \widetilde{F}_{2}\left(L_{3}\right)$ (onto). Define $F_{2}=\Psi^{-1} \circ \widetilde{F}_{2}$ on $L_{3}$ and $F_{2}=\left.\Psi\right|_{L_{2}}$ on $L_{2}$. We take $r_{2}$ so close to $r_{1}$ that $\Psi\left(L_{2}\right) \supset \mathrm{cl} F_{2}\left(L_{4}\right)$.

Now take $V=L_{2} \cup L_{3} \cup L_{4}$ and $F_{2}$ defined on $V$ as above.

The assumptions of Lemma 2.1 are satisfied. So we can "glue" $F_{1}$ and $F_{2}$ in one rational mapping $f$.
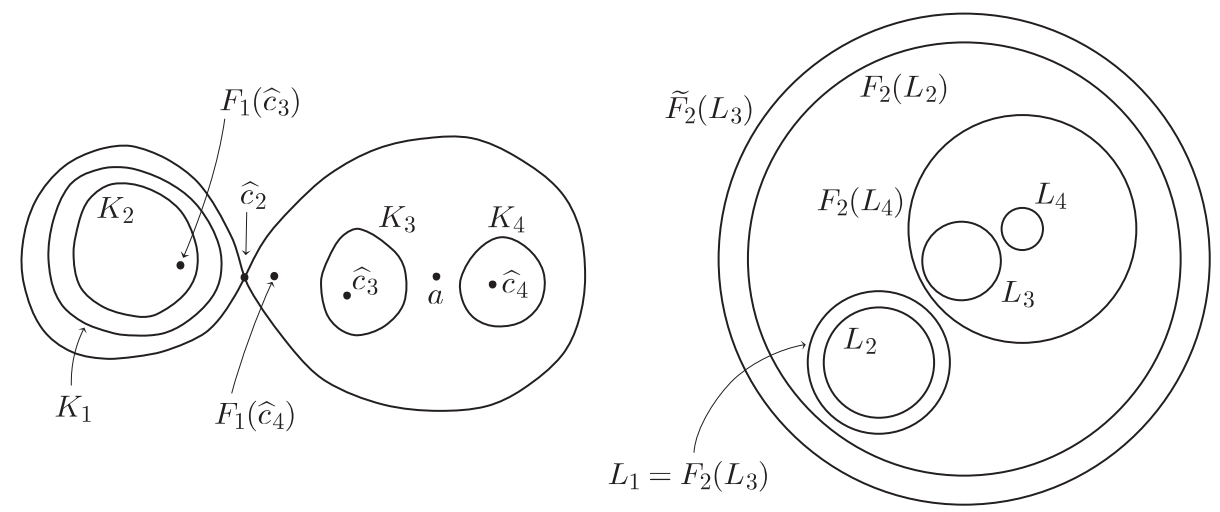

Fig. 6

Observe finally that $J(f)$ is disconnected because $F_{1}^{n}\left(\widehat{c}_{2}\right) \rightarrow \infty$ and moreover $F_{1}^{n}(l) \rightarrow \infty$, where $l=\left\{\Im z=\Im \widehat{c}_{2}\right\}$ ( $\Im$ denotes imaginary part). The line $l$ separates $K_{2}$ from say $K_{4}$. Both $K_{1}$ and $K_{4}$ intersect $J(f)$ (in the coordinates after the integration of $\mu_{1}$ ) so the intersections belong to different components of $J(f)$.

The degree of $f$ on the basin of attraction to $\infty$ is 3 because such is the degree of $F_{1}$ on $U$. Only two critical points: $\infty$ and the one corresponding to $\widehat{c}_{2}$ belong to the basin, because $\widehat{c}_{3,4}$ do not escape under iteration by $F_{2}$. Theorem $\mathrm{D}$ is thus proved for $d=3$. 
R e mark 2.2. Observe that in appropriate holomorphic coordinates on $\overline{\mathbb{C}}$ we have $f(z)=z^{2}+c+b /(z-a)$. Indeed, after subtracting from the $f$ constructed above the principal part of the Laurent series expansion at the pole, we are left with a quadratic polynomial. By an affine holomorphic change of coordinates we make the polynomial be $z^{2}+c$.

R e mark 2.3. One should be careful in the above construction because not every branched cover of $\overline{\mathbb{C}}$ preserves a conformal structure. Above, an annulus $\mathcal{A}$ in $B_{2}$ containing $c_{3}$ and $c_{4}$ is mapped in a proper way by $f$ to the disc $D^{\prime}=\left\{G<t^{\prime}\right\}, t^{\prime}>t$, i.e. a disc in $\overline{\mathbb{C}}$ bounded by level $t^{\prime}$ of Green's function $G$, outside the figure 8 level $\{G=t\}$.

Instead of mapping $c_{3}$ into $D_{1}$ so that $F^{2}\left(c_{3}\right)=c_{3}$ we can map $\mathcal{A}$ onto $D^{\prime}$ in a proper way so that $f\left(c_{3}\right)=c_{3}$ and $f\left(c_{4}\right)=c_{4}$. This will be a topological branched cover. However, it does not admit a holomorphic invariant structure.

Indeed, for a holomorphic $f$, either $f^{n}\left(c_{3}\right)$ or $f^{n}\left(c_{4}\right)$ must escape from $\mathcal{A}$. Otherwise we consider the branch $g$ of $f^{-1}$ on $D^{\prime} \backslash \mathcal{A}$ mapping $\partial D^{\prime}$ to the external component of $\partial \mathcal{A}$. All moduli of the annuli $P_{n}:=g^{n}\left(D^{\prime} \backslash \mathcal{A}\right)$ are the same, and positive, so $\bmod \bigcup_{n} P_{n}=\infty$ and hence $D^{\prime} \backslash \bigcup_{n} P_{n}$ consists of only one point and contains $c_{3} \neq c_{4}$, a contradiction $\left({ }^{4}\right)$.

Another argument is that such an $f$ would have 3 superattracting fixed points. So it would be a Newton's method rational function of a degree 3 polynomial (see the introduction). But the basin of attraction to $\infty$ is not simply connected. This contradicts a theorem that the basins of immediate attraction to the attracting fixed points for Newton's method are simply connected $[\mathrm{P}]$.

Proof of Theorem D, the general case $d \geq 3$. We shall realize holomorphically the picture of Fig. 7:

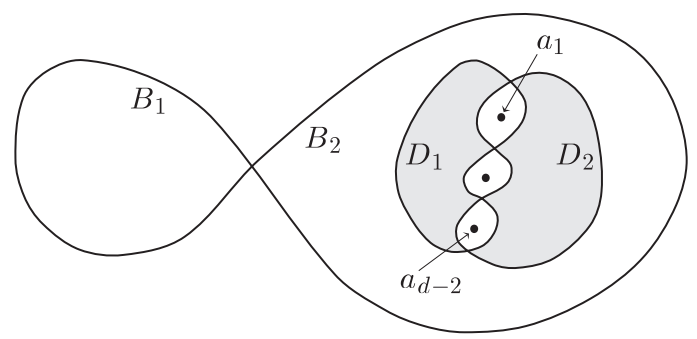

Fig. 7

In Fig. $7, D_{j}$ is mapped properly onto $B_{j}$ for $j=1,2$. Each $D_{j}$ contains $d-2$ critical points. The points $a_{1}, \ldots, a_{d-2}$ are poles.

$\left({ }^{4}\right)$ This proof was obtained in a discussion with K. Barański after the preprint version of this paper appeared. It will appear in a wider context in $[\mathrm{Ba}]$. 
We proceed similarly to the case $d=3$. Let

$$
F_{1}(z)=z^{2}+c+b \sum_{m=1}^{d-2} \frac{1}{z-a_{m}} .
$$

Take $a_{m}=\sqrt{-c}+i m T$ for a real constant $T$ with $0<T \ll 1$, in particular $T$ small enough that all $a_{m}$ are well in $B_{2}$.

For $b>0$ small, there is a small annulus around each pole $a_{m}$, containing two critical points

$$
\widehat{c}_{m, 3}, \widehat{c}_{m, 4}=a_{m} \mp \sqrt{b /\left(2 a_{m}\right)}+o(\sqrt{b}) .
$$

The corresponding critical values

$$
v_{m, 3}=F_{1}\left(\widehat{c}_{m, 3}\right), \quad v_{m, 4}=F_{1}\left(\widehat{c}_{m, 4}\right)
$$

are

$a_{m}^{2}+c \mp 2 \sqrt{2 a_{m}} \sqrt{b}+o(\sqrt{b})=2 i m T \sqrt{-c}+\left(-m^{2} T^{2} \mp 2 \sqrt{2 a_{m}} \sqrt{b}+o(\sqrt{b})\right)$.

(Computing $\widehat{c}_{m, 3(4)}$ and $v_{m, 3(4)}$ we need only consider $z^{2}+c+b /\left(z-a_{m}\right)$. Other terms $b /\left(z-a_{t}\right)$ make only an $O(b)$ contribution.)

The $F_{1}$-critical point close to 0 is $O(b)$.

Take $K_{1}, K_{4}^{\prime}$ from the case $d=3$ slightly modified, larger than the original ones: Let $l$ be the line (parabola) $\left(2 i T \sqrt{-c} \tau,-T^{2} \tau^{2}\right)$ for $\tau>0$. Observe that the critical values $v_{m, 3}$ are to the left of $l$, and $v_{m, 4}$ to the right of $l$. We extend $K_{1}, K_{4}^{\prime}$ to $\widehat{K}_{1}, \widehat{K}_{4}^{\prime}$ almost reaching $l$ to capture $v_{m, 3}, v_{m, 4}$ respectively (see Fig. 8).

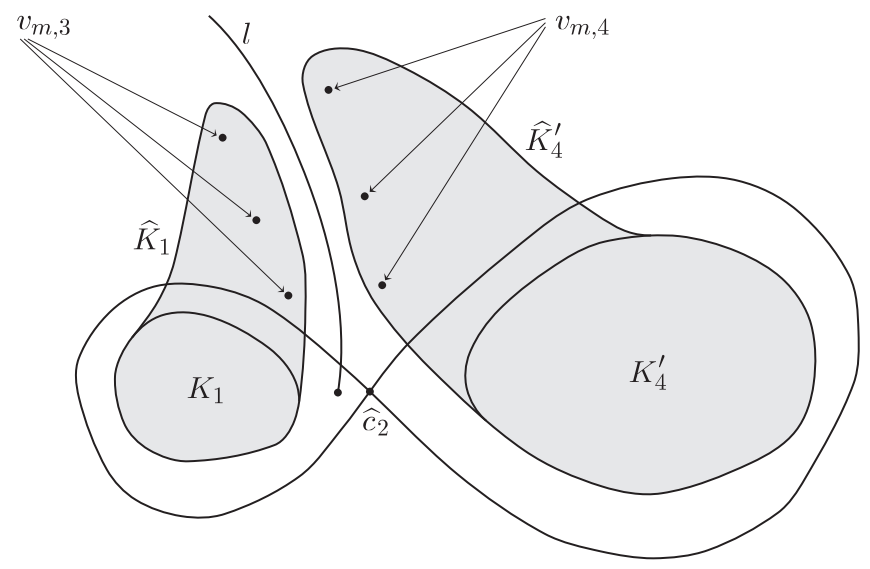

Fig. 8

Consider the topological discs

$$
K_{m, 3}=\operatorname{Comp} F_{1}^{-1}\left(K_{1}\right), \quad K_{m, 4}=\operatorname{Comp} F_{1}^{-1}\left(K_{4}^{\prime}\right),
$$


where Comp means the component containing $\widehat{c}_{m, 3}, \widehat{c}_{m, 4}$ respectively. Let $K_{2}$ be as in the case $d=3$, such that $F_{1}\left(K_{2}\right) \supset \operatorname{cl} K_{4}^{\prime}$. Finally, set

$$
U:=\overline{\mathbb{C}} \backslash\left(K_{2} \cup \bigcup_{m=1}^{d-2}\left(K_{m, 3} \cup K_{m, 4}\right)\right) .
$$

The rest of the construction of $f$ is the same as for $d=3$. When we define quadratic-like maps $\widetilde{F}_{2}$ on $L_{m, 3}$ and $F_{2}$ on $L_{m, 4}$ we have a complete freedom of choosing quadratic polynomials we glue in, in particular, we can have the corresponding critical points escaping or not. (In particular, if no critical point escapes we have the most surprising case $k=2$ of the assertion of Theorem D.)

For completeness of exposition we prove the following simple facts (the first of them was already stated in the introduction):

Proposition 2.4. (a) Let $f \in H^{d}$ be a polynomial with $B(f)$, the basin of attraction to $\infty$, not simply connected. Then $B(f)$ contains at least one critical point different from $\infty$.

(b) More generally, if $f \in Q^{d}$ and for a pair of topological discs $A, A_{1}$ with $\operatorname{cl} A \subset A_{1}$ the map $\left.f\right|_{A}: A_{1} \rightarrow A$ is proper of degree $d^{\prime} \leq d$, then $A_{1}$ is in the basin of attraction $B(f)$ to an attracting fixed point, and if $B(f)$ is not simply connected then it contains at least $d^{\prime}$ critical points. This concerns in particular the case $d^{\prime}=d$ in which $\left.f\right|_{\overline{\mathbb{C}} \backslash \mathrm{cl} A_{1}}: \overline{\mathbb{C}} \backslash \operatorname{cl} A_{1} \rightarrow \overline{\mathbb{C}} \backslash \operatorname{cl} A$ is polynomial-like.

Proof. (a) Take a topological disc $D=\{G>a\}$ around $\infty$ (cf. proof of Corollary B or Remarks 1.5, 1.6). If there are no critical points in $B(f)$ (except $\infty$ ) then $f^{-n}(D)$ is an increasing sequence of topological discs, so $B(f)=\bigcup_{n \geq 0} f^{-n}(D)$ is a topological disc, and hence $B(f)$ is simply connected.

(Note that in Remark 2.2 we already used the argument that if there is only one critical value for a proper map $f: W_{1} \rightarrow W_{2}$ where $W_{2}$ is a topological disc, then $W_{1}$ is also a topological disc.)

(b) The proof is similar. There are $d^{\prime}-1$ critical points in $A_{1}$ and there must be one in $B(f) \backslash A_{1}$.

(One can also deduce (b) from (a) using a Blaschke type perturbation, Section 1.)

Proposition 2.5. Every non-simply connected immediate basin of attraction to an attracting or parabolic fixed point $p\left(\right.$ with $\left.f^{\prime}(p)=1\right)$ contains at least 2 different critical values for critical points in the basin.

This complements Theorem D: the integer $k$ cannot be less than 2 . 
Proof. Consider the sets $D_{n}$ defined in Remark 1.6. As $B(f)=$ $\bigcup_{n \geq 0} D_{n}$ is not simply connected, there exists $n$ such that $D_{n}$ is simply connected and $D_{n+1}$ is not. Then $D_{n}$ contains at least two different critical values for critical points in $D_{n+1}$.

(Almost the same argument proves the above for periodic basins, with period larger than 1.)

Proof of Corollary E. Let $f \in H^{d}$ be as in Theorem $\mathrm{D}$ for $d \geq$ $3, k=2$. Consider an arbitrary $g \in H(f)$. Then there exist a real continuous 1-parameter family of homeomorphisms $h_{t}: \overline{\mathbb{C}} \rightarrow \overline{\mathbb{C}}$ and a real 1-parameter family of maps $f_{t} \in H(f)$ having the same properties as $h_{\lambda}$ and $f_{\lambda}$ in Remark 1.4. (There exist complex families precisely as in Remark 1.4, but we do not need them here.) Then $\sharp\left(\operatorname{Crit}\left(f_{t}\right) \cap B\left(f_{t}\right)\right)$ is constant because critical points cannot be too close to $J(t)$, where $\left|f^{\prime}\right|>0$ uniformly, and they move continuously with $t$, so they cannot jump between components of $\overline{\mathbb{C}} \backslash J\left(f_{t}\right)$. So by Proposition 2.4, $B(g)$ cannot be the basin of attraction to $\infty$ for a polynomial. But the degree of $f$, and hence of $g$, on any other invariant basin $B_{1}$ is less than $d$. (Otherwise $\partial B_{1}=J(f)$ would be connected and it is not because $B(f)$ is not simply connected.) So $g$ cannot be a polynomial.

Note that it follows from Proposition 2.4(b) and the above proof that no $g \in H(f)$ has a polynomial-like restriction of degree $d$.

3. A 1-parameter family of functions joining an exotic $z \mapsto$ $z^{2}+c+b /(z-a)$ to a Newton's method rational function. Let $f(z)=$ $z^{2}+c+b /(z-a)$. Then $f^{\prime}(z)=2 z-b /(z-a)^{2}$. The equation for the critical points in $\overline{\mathbb{C}}$ is

$$
2 z(z-a)^{2}=b
$$

Suppose that $w=c_{4}$ is an $f$-fixed critical point (see Fig. 5, Section 2). (This restricts the number of parameters to 2.) We obtain

$$
\left(w^{2}-w+c\right)(w-a)=-b, \quad 2 w(w-a)^{2}=b .
$$

Let $a=k w$. We parametrize $f$ by $k$ and $w$. We obtain

$$
a=k w, \quad b=2 w^{3}(1-k)^{2}, \quad c=w^{2}(2 k-3)+w .
$$

The critical points are $u=c_{2}, v=c_{3}, w$, where

$$
u, v=w\left(-\frac{1}{2}+k \mp \frac{1}{2} \sqrt{4 k-3}\right) .
$$

Given a parameter $k<1$ sufficiently close to 1 , one finds $w$ such that $f^{2}(v)=v$ and the trajectory of $u$ escapes to $\infty$. For $k=.85$ one finds $w \approx 1.88053$. This is an exotic example as in Fig. 5, Section 2. The reason is that the geometry is as in Fig. 6, Section 2, so the basin $B$ of attraction 
to $\infty$ is connected, i.e. the immediate basin is completely invariant. The picture is similar to that in Fig. 10d.

(It is not clear to me whether just the escape of $u$ to $\infty$ proves the connectedness of $B$. One should be careful because for $f$ being only a topological branched cover this is not so; see the example in Remark 2.3.)

In the rest of this section we discuss the change of dynamics for varying parameter $w$.

For $k=.85$ the number $w=1.88053$ is in the principal part of a Mandelbrot-like set $M\left(c_{3}\right)$, symmetric with respect to real $w$ 's, pronged to the left. For $w \in M\left(c_{3}\right)$, the Julia set for quadratic-like $\left.f^{2}\right|_{D_{1}}$ (see Fig. 5 , Section 2) is connected and we still have exotic maps.

Now let us decrease $w$. It leaves $M\left(c_{3}\right)$ at $w \approx 1.86874$ and below that $w$ the trajectory of $v$ escapes from $D_{1}$. It need not escape to $\infty$. There is a sequence of intervals where $f^{2 n}(v)$ hits $B_{w}$, the basin of immediate attraction to $w$, as $n$ decreases to 2 . Later on, after escape, again $f^{4}(v) \in B_{w}$ but $f^{2}(v)<u$ (before, it was between $w$ and $v$ ). This happens at $w \approx 1.63045$ (see Fig. 9).

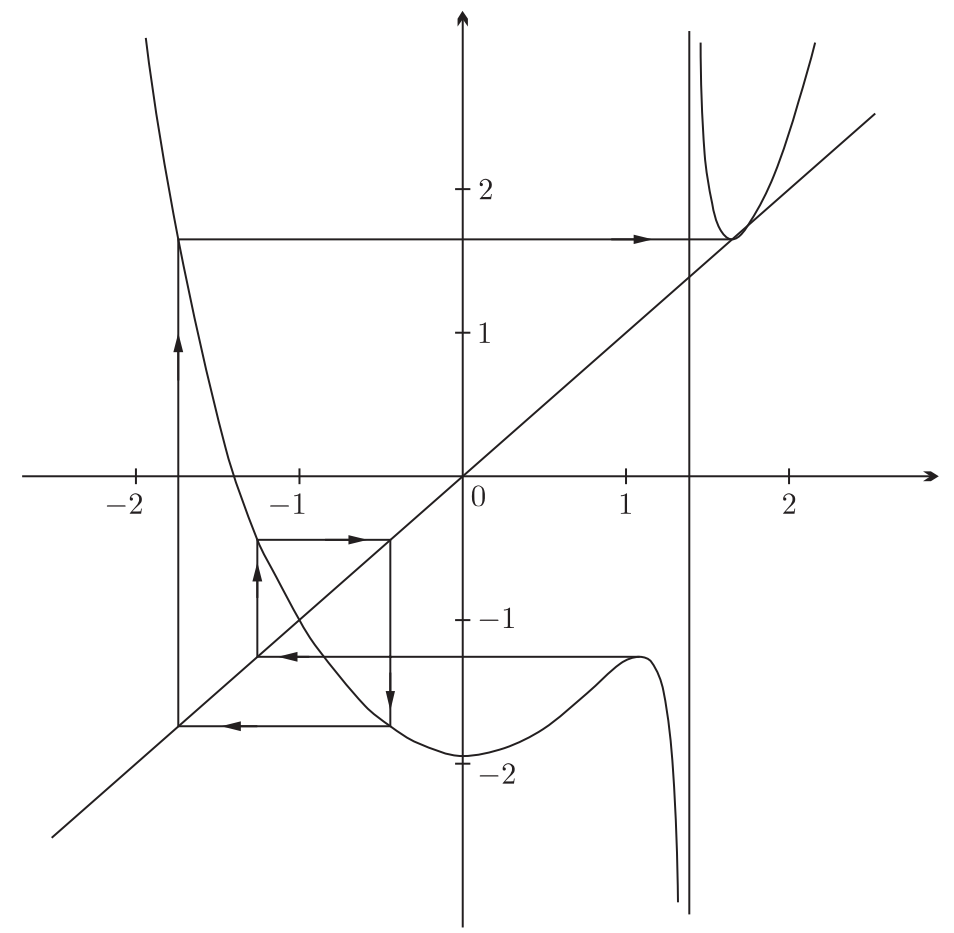

Fig. 9. $k=.85, w=1.63045$

At some parameter $w$ the trajectory of $u=c_{2}$ stops to escape to $\infty$. It hits $B_{w}$. But next with further decrease of $w$ it can again escape to $\infty$. 
Starting from $w \approx 1.541549$ the trajectory of $u$ neither escapes to $\infty$ nor to $w$. The parameter $w$ is in a Mandelbrot-like set $M\left(c_{2}\right)$ pronged towards the right. In fact, at this parameter $f^{2}(u) \in(v, a)$. Only after some further decrease of $w$ do we arrive at $f^{2}(u) \in(u, v)$, so that one has a unimodal map $f:(f(u), v) \mapsto(f(u), v)$.

$w \approx .713615$ is in the principal part of $M\left(c_{2}\right)$ and $f$ is Newton's. Then $f^{n}\left(c_{3}\right) \rightarrow c_{2}=f\left(c_{2}\right)$. The number $w \approx .303151$ is still in the principal part of $M\left(c_{2}\right)$ and $f$ is Newton's but now $f^{n}\left(c_{2}\right) \rightarrow c_{3}=c_{3}\left({ }^{5}\right)$.

Let us now present pictures from this experiment for $k=.81\left({ }^{6}\right)$.

In Fig. $10, k=.81$, white is the basin of attraction to $w$, grey the basin of $\infty$, black is the complement. For Newton's $f$ (Fig. 10a), black contains both $c_{2}$ and $c_{3}$, so it has a connected interior and approaches the only repelling fixed point in two channels. Let $w$ grow. For $w \approx 1.37$ the black Newton's basin has bifurcated to period 4, Fig. 10b.

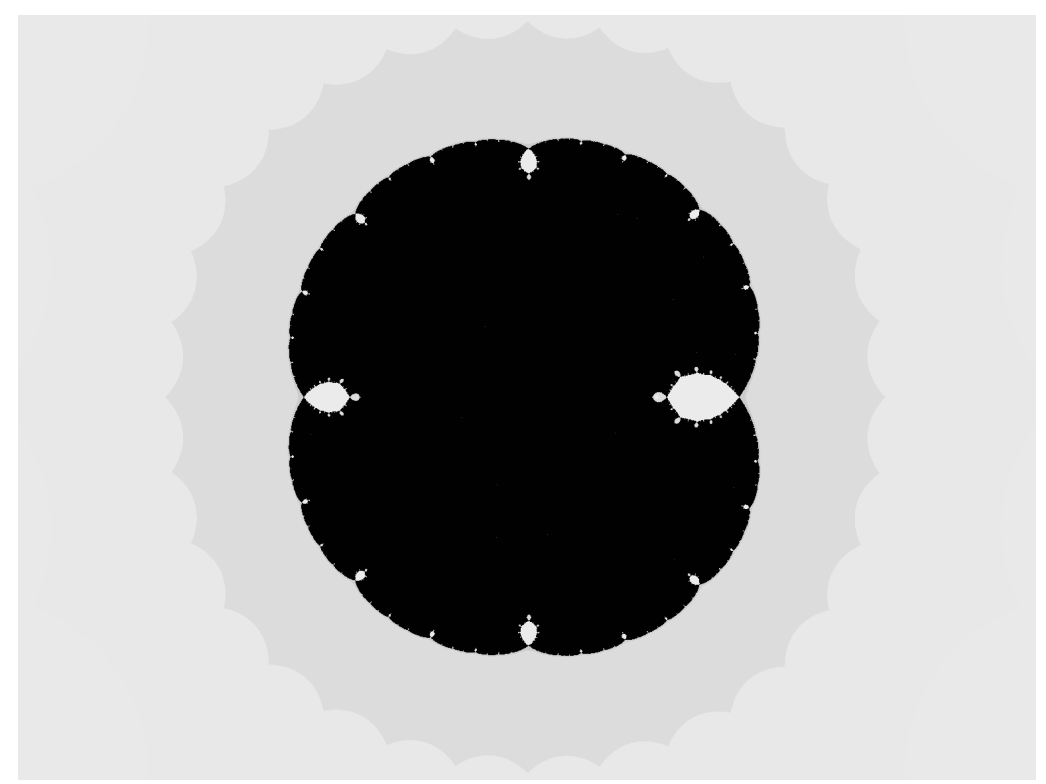

Fig. 10a. $k=.81, w=.63$, window $-2-2 i, 2+2 i$. Iteration of a Newton's method rational map for a polynomial. Black, white and grey are basins of attraction to the zeros of the polynomial.

$\left({ }^{5}\right)$ This description comes from a computer made picture in 9 colors, showing whether $c_{2}, c_{3}$ escape to $\infty$, to $w$ or behave differently.

$\left({ }^{6}\right)$ Added July 1995: I received recently a letter from Prof. Kiyoko Nishizawa (Josai University, Japan) containing the description of a variety of Newton's maps. I slightly corrected the estimated values of the Newton's parameters $w$ for $k=.85$ according to his computations. 


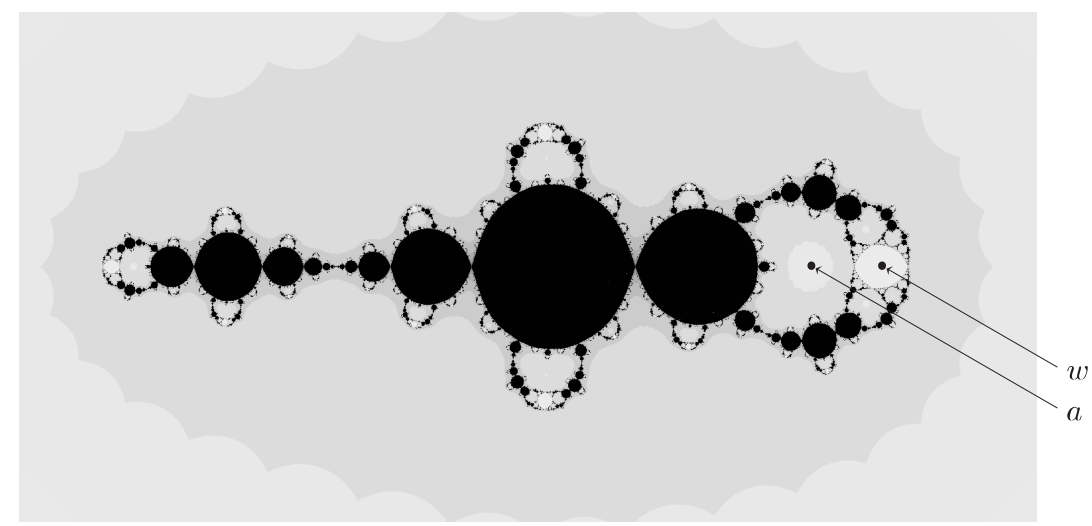

Fig. 10b. $k=.81, w=1.37$, window $-2-2 i, 2+2 i$. The black Newton's basin has bifurcated to a period 4 immediate basin and its preimages.

For $w \approx 1.4961$ (Fig. 10c), $w$ is already in $M\left(c_{3}\right)$ but $u=c_{2}$ does not escape to $\infty$. It is in the basin of $w$. The basin of $\infty$ is not connected. This is so because the immediate basin (and the whole basin too) contains only 1 critical point: $\infty$. So it is simply connected (see Proposition 2.5). Hence $f$ has only degree 2 on this immediate basin.

For $w \approx 1.51545, u$ escapes to $\infty$. The basin of $\infty$ becomes connected. This is one of our exotic examples: see Fig. 10d.

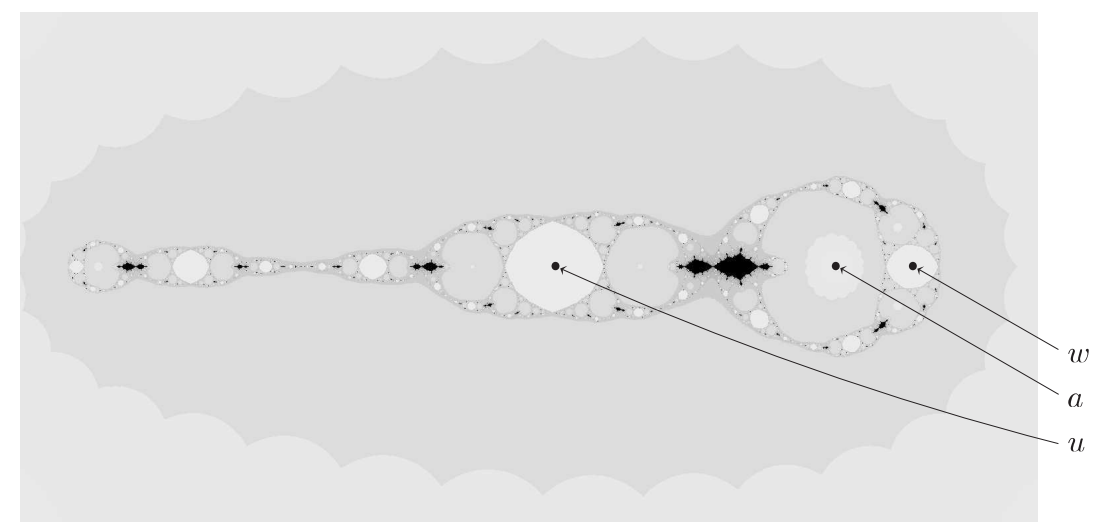

Fig. 10c. $k=.81, w=1.49$, window $-2-2 i, 2+2 i$. The map is still not exotic because the trajectory of the critical point $u$ is attracted to $w$.

QUESTION 3.1. In the set of Newton's method rational functions $N P_{\lambda}$ for the polynomials $P_{\lambda}(z)=z^{3}+(\lambda-1) z-\lambda$ there exist Mandelbrot-like sets of "exotic" parameters for which the critical point different from the zeros of $P_{\lambda}$ converges to a periodic attracting orbit different from these zeros 


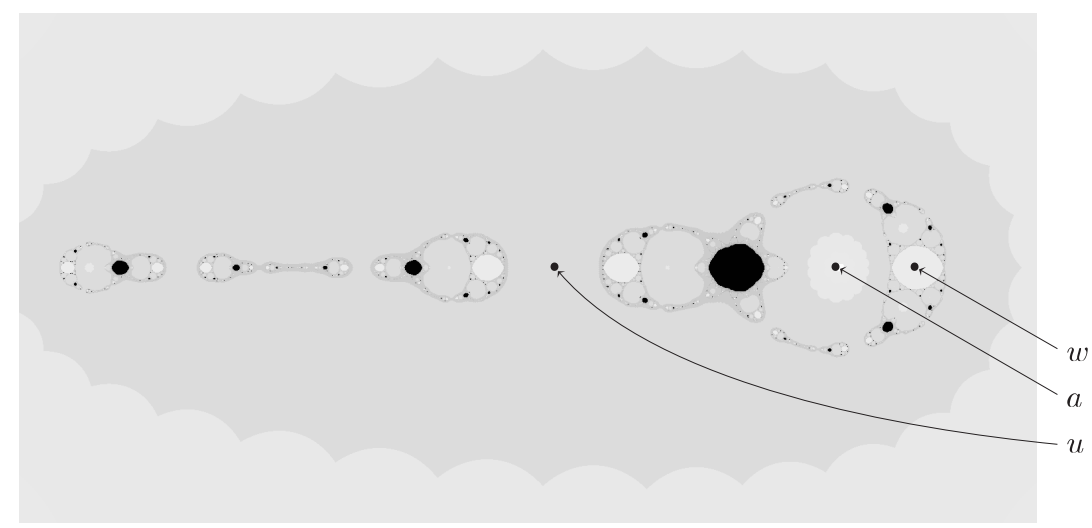

Fig. 10d. $k=.81, w=1.51545$, window $-i, 2+i$. This is an exotic map. The pattern is as in Figs. 5, 6. The union of black and white does not separate the plane any more, $u$ escapes to $\infty$.

[CGS]. Do these sets move to $M\left(c_{3}\right)$ sections of the set of our exotic maps when we change parameters from Newton's to our exotic ones $\left({ }^{7}\right)$ ?

Question 3.2. Describe precisely how the dynamics bifurcates (i.e. describe the limit behaviour of the trajectories of $c_{2}$ and $c_{3}$ ) for real parameters $k, w$. This question concerns the iteration of a real map having 2 critical points, namely our $f$ restricted to $(-\infty, a)$. (The right branch from $a$ to $\infty$ does not take part in the recurrence because for $z>a$ and every $n \geq 0$, $f^{n}(z) \geq a$.)

\section{References}

[AB] L. Ahlfors and L. Bers, Riemann's mapping theorem for variable metrics, Ann. of Math. 72 (1960), 385-404.

[Ba] K. Barański, PhD thesis, in preparation.

[B] B. Bojarski, Generalized solutions of systems of differential equations of first order and elliptic type with discontinuous coefficients, Mat. Sb. 43 (85) (1957), 451-503 (in Russian).

[Boy] M. Boyle, a letter.

[CGS] J. Curry, L. Garnett and D. Sullivan, On the iteration of a rational function: computer experiments with Newton's method, Comm. Math. Phys. 91 (1983), 267-277.

[D] A. Douady, Chirurgie sur les applications holomorphes, in: Proc. ICM Berkeley 1986, 724-738.

$\left({ }^{7}\right)$ Added July 1995: One can find a family $f_{\mu, \lambda}$ of rational maps of degree $3, \mu \in$ $(0,1)$, so that for each $\mu<1 / 2$ the maps $f_{\mu, \lambda}$ are Newton's "exotic" (or in their hyperbolic component), and for $\mu>1 / 2$ our exotic. See [Ba]. 
[DH1] A. Douady and J. Hubbard, On the dynamics of polynomial-like mappings, Ann. Sci. École Norm. Sup. 18 (1985), 287-243.

[DH2] —, Étude dynamique des polynômes complexes, Publ. Math. Orsay 2 (1984), 4 (1985).

[GK] L. Goldberg and L. Keen, The mapping class group of a generic quadratic rational map and automorphisms of the 2-shift, Invent. Math. 101 (1990), 335372.

[M] P. Makienko, Pinching and plumbing deformations of quadratic rational maps, preprint, Internat. Centre Theoret. Phys., Miramare-Trieste, 1993.

[P] F. Przytycki, Remarks on simple-connectedness of basins of sinks for iterations of rational maps, in: Banach Center Publ. 23, PWN, 1989, 229-235.

INSTITUTE OF MATHEMATICS

POLISH ACADEMY OF SCIENCES

ŚNIADECKICH 8

00-950 WARSZAWA, POLAND

Received 7 April 1994;

in revised form 14 July 1995 